\title{
Balloon Dilatation for an Esophageal Stricture by Long-Term Use of a Nasogastric Tube: A Case Report
}

\author{
Yong-Soon Yoon, MD, $\mathrm{PhD}^{1}$, Jong Yun Kim, $\mathrm{MD}^{1}$, Kwang Jae Lee, MD, $\mathrm{PhD}^{1}$,
} Ki Pi Yu, MD ${ }^{1}$, Mi Sook Lee, MD $^{2}$

Departments of ${ }^{1}$ Rehabilitation Medicine and ${ }^{2}$ Radiology, Presbyterian Medical Center, Jeonju, Korea

In the present report, we describe a case of long-term follow-up esophageal stricture occurring in a patient with nasogastric tube use. A 63-year-old man who had experienced dislocation of the 6th and 7th cervical vertebrae as the result of an external injury received treatment at another hospital and was admitted to the rehabilitation department of our hospital. After he exhibited normal swallowing in a videofluoroscopic swallowing test, the nasogastric tube was removed and oral feeding with a dysphagia diet was initiated. However, during oral feeding, the patient complained of swallowing difficulties in his lower throat. An esophagogastroduodenoscopy was performed to examine the lesions below the pharynx and a 2-mm stricture was observed. A balloon dilatation was performed for a total of 9 times to extend the stricture. After the procedure, the patient was able to easily swallow a normal diet through the esophagus and the vomiting symptoms disappeared. An esophagography showed that the diameter of the esophageal stricture was $11 \mathrm{~mm}$.

Keywords Nasogastric tube, Esophageal stricture, Ballooning dilatation

\section{INTRODUCTION}

Enteral nutrition is defined as the administration of complete nutritional preparations into the stomach either orally or by using tubes [1]. Tube feeding should be considered when a patient cannot orally consume food but has a normally functioning gut. Although there are

Received August 5, 2013; Accepted October 4, 2013

Corresponding author: Jong Yun Kim

Department of Rehabilitation Medicine, Presbyterian Medical Center, 365, Seowon-ro, Wansan-gu, Jeonju 560-750, Korea

Tel: +82-63-230-1460, Fax: +82-63-282-3385, E-mail: kijoyu@hanmail.net

(c) This is an open-access article distributed under the terms of the Creative Commons Attribution Non-Commercial License (http://creativecommons. org/licenses/by-nc/3.0) which permits unrestricted noncommercial use, distribution, and reproduction in any medium, provided the original work is properly cited.

Copyright $\odot 2014$ by Korean Academy of Rehabilitation Medicine several kinds of feeding tubes, nasogastric (NG) tubes are commonly used, because they can be easily placed without the need for surgery. However, gastrointestinal, respiratory, and metabolic problems can arise from artificial feeding through an NG tube [2]. Because multiple complications may occur during the long-term use of NG tubes, it is necessary to change to other methods with fewer complications, such as gastrostomy or enterostomy, when tube feeding will be required for more than 3 to 4 weeks [3].

Esophageal strictures associated with the use of NG tubes have rarely been reported in the literature in other countries [4,5]. In Korea, case reports have described balloon dilatation of esophageal strictures that originate from other causes than the use of NG tubes [6,7]. In the present report, we describe a case of esophageal stricture 
developing after the long-term use of a NG tube in a patient who was admitted to the department of rehabilitation.

\section{CASE REPORT}

A 63-year-old man presented to the emergency room of another hospital with muscle weakness of the limbs after a cow struck his neck and chest. Magnetic resonance imaging of the cervical spine performed at that time revealed a dislocation of the 6 th and 7 th cervical vertebrae. An anterior internal fixation was conducted from the 6th cervical vertebra to the 1 st thoracic vertebra on the day of the injury and a conservative treatment that involved maintaining a supine position was recommended. The patient was admitted to our hospital for rehabilitation treatment 99 days after the injury. The neurological level of injury was determined to be $\mathrm{C} 5$ according to the American Spinal Injury Association Impairment scale B. At that time, the patient was using an NG tube. A videofluoroscopic swallowing (VFS) test was attempted at 1 week after admission, but the test could not be performed because the patient could not remain in the required position. Hence, the test was performed 128 days after injury, after confirming that the patient could maintain the required position for the procedure. The oropharyngeal examination during the VFS test showed that the swallowing reflex and processes had progressed without any abnormal findings; therefore, the NG tube was removed

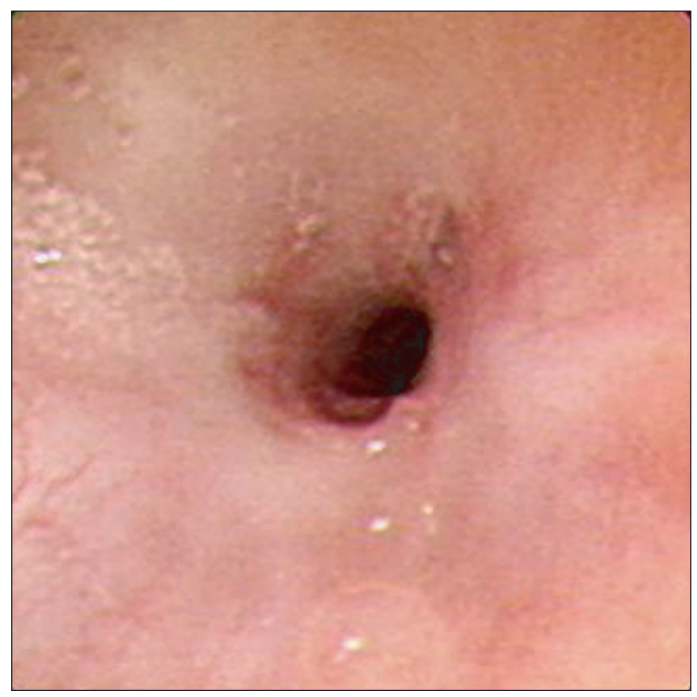

Fig. 1. Esophagogastroduodenoscopy: a stricture is visible $35 \mathrm{~cm}$ below the inferior pharyngeal constrictor muscle. and the oral feeding of a dysphagia diet was initiated.

The patient complained of swallowing during the feeding because of food stuck in the throat; moreover, he experienced intermittent vomiting after the feeding. The ability to swallow at that time was estimated to be of grade 2 on the Functional Oral Intake Scale (FOIS) [8]. A laryngoscopy performed 3 days prior to the occurrence of symptoms revealed no abnormal findings like as laryngeal swelling. First, the patient underwent a surgery to treat a concomitant complaint of sore coccyx. Subsequently, an esophagogastroduodenoscopy (EGD) was performed 27 days after the initiation of oral feeding. This EGD indicated a stricture at $35 \mathrm{~cm}$ below the inferior pharyngeal constrictor muscle and was unable to reach beyond this point (Fig. 1). The patient had no history of dysphagia prior to his injury, received no medications inducing endoscopy or stenosis before or after the injury and had no history of medical irradiation. Therefore, his stricture was suspected to be related to the NG tube. The patient was kept in a sitting position of $45^{\circ}$ and antiulcer medication was added. The following day, a computed tomography of the chest was performed to examine a transverse section of the esophageal stricture. No mediastinal mass was
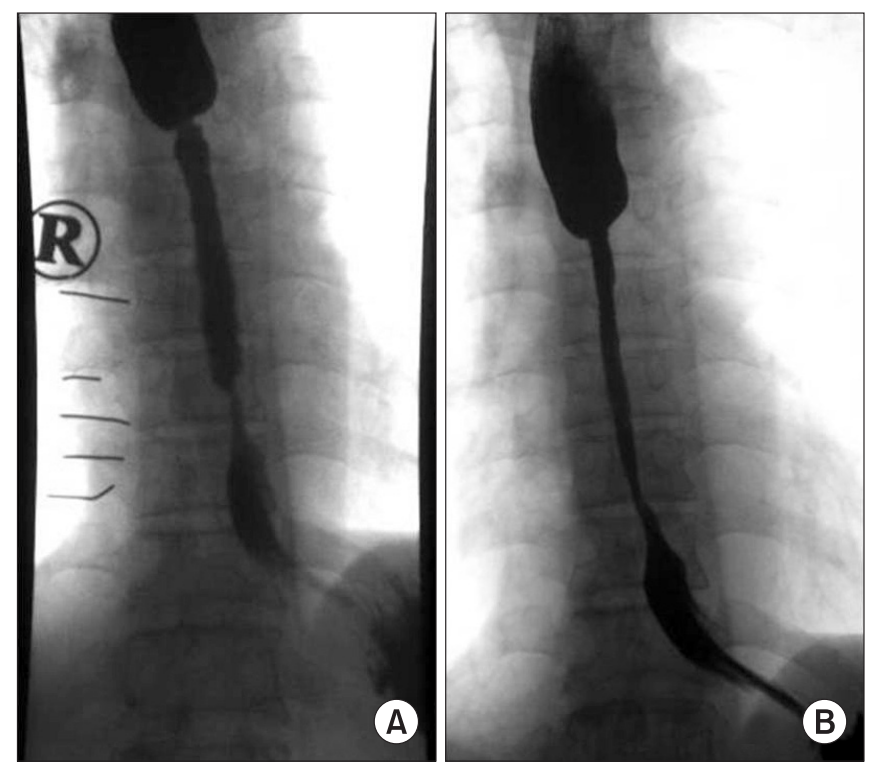

Fig. 2. (A) Esophagogram before balloon dilatation: the stricture lumen is approximately $0.2 \mathrm{~cm}$ in length. (B) After 4 applications of balloon dilatation, a slight dilatation is observed and the stricture lumen is approximately $0.5 \mathrm{~cm}$ in length. The drainage of the contrast medium is improved, but the stricture within the mid-esophagus persists. 
observed, but a diffuse submucosal edema was present at the left, upper lobar bronchus level of the esophagus and the lumen was narrowed. A balloon dilatation was performed to extend the stricture. EGD-guided insertions of balloon catheters were performed; twice at $1 \mathrm{kPa}$ for 1 minute and twice at $2 \mathrm{kPa}$ for 1 minute, thus achieving a dilation of approximately $7 \mathrm{~mm}$. The resulting esophagogram revealed an increase of the stricture lumen diameter by $3 \mathrm{~mm}$. The patient's symptoms improved, but recurred after 1 day; therefore, a follow-up esophagography was performed. The procedure revealed that the diameter of the stricture had increased after the procedure, but the stenosis still remained. Therefore, an esophageal resection and a reconstruction with the left colon were recommended. However, the patient and his family consented to the surgery, especially and particularly because it would be considered as high risk due to his history of flail chest. A high risk of postoperative complications was also expected. Thus, the balloon dilatation was performed for a total of 4 times at our hospital. After the last balloon dilatation procedure, the patient's symptoms improved to grade 4 on the FOIS scale and an extension of 5 $\mathrm{mm}$ was observed for the lumen of the esophageal stricture by esophagography (Fig. 2). After discharge from our hospital, the patient regularly received balloon dilatation at another hospital for a total of 5 times. Each of the dilations was done for one minute for two times, similarly to the procedure in our hospital. Currently, his esophageal stricture is extended to $11 \mathrm{~mm}$ in diameter and the swallowing symptoms he experiences during meals have im-

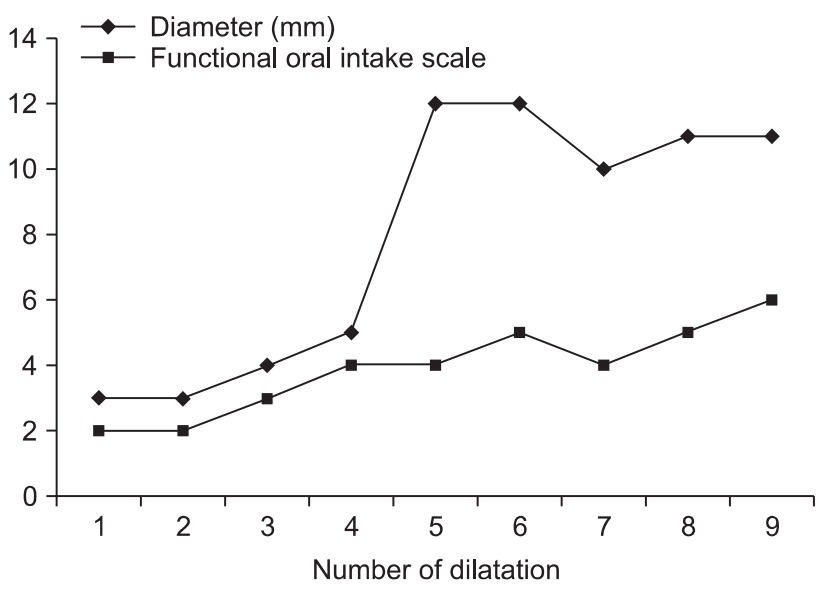

Fig. 3. Follow-up with the Functional Oral Intake Scale, and stricture diameter after repeated ballooning dilatations. proved to grade 6 on the FOIS scale (Fig. 3).

\section{DISCUSSION}

The etiology or pathogenesis of esophageal stricture associated with NG tubes has not yet been fully elucidated. In particular, many studies have addressed the role of gastroesophageal reflux in such cases. Nagler et al. [9] suggest that the esophageal acid clearance is delayed in subjects lying in the supine position because of the lack of gravitational components acting on the esophageal emptying mechanisms. After being transferred to our hospital, the patient in the present case was predominantly maintained in the supine position until the rehabilitation treatment was completed. This appears to have increased the frequency of gastroesophageal reflux and thereby caused the stricture.

When the patient was diagnosed with esophageal stricture, a series of conservative treatment was provided. The patient had to change his posture and got more medication. However, it had been reported that such treatment has an insignificant effect [4]. The unresponsiveness of an esophageal stricture to conservative treatments, such as medication, is a severe complication that requires intervention. The balloon dilatation is the most common initial treatment for NG tube-related esophageal strictures. Its success rate is dependent upon the stricture length [8]. Patients with a short stricture length in whom a satisfactory dilatation can be achieved can be treated with dilatation only. However, when a stricture is long and does not respond to dilatation, it is strongly advisable to proceed with an esophageal resection and a left colon interposition. At present, after 9 dilatations, the patient's esophageal diameter improved to $11 \mathrm{~mm}$ and his state improved to grade 6 on the FOIS. However, Egan et al. [10] reported that dilations of the esophageal diameter from 1.3 to $2.0 \mathrm{~mm}$ can provide relief in $85 \%-93 \%$ of patients with dysphagia. However, this study was conducted in the United States based on the reference data of this country and these results may differ in Korean individuals due to the different body types and dietary habits. To address this issue, we believe that further study is necessary to investigate the results of long-term follow-up.

In conclusion, we reported a case of long-term followup esophageal stricture that occurred after the use of a NG tube. Although there is only a rare occurrence of 
esophageal stricture complications, its severity is remarkably high. Therefore, the maintenance of a sitting position and the administration of preventive ulcer drugs are necessary to prevent the esophageal reflux in patients with NG tube feeding. Moreover, the duration of a NG tube placement should be limited to 1 month. If this is not feasible, we recommend changing the method of feeding to a gastrostomy in accordance to the appropriate clinical judgment.

\section{CONFLICT OF INTEREST}

No potential conflict of interest relevant to this article was reported.

\section{REFERENCES}

1. Kirby DF, Delegge MH, Fleming CR. American Gastroenterological Association technical review on tube feeding for enteral nutrition. Gastroenterology 1995;108:1282-301.

2. Pancorbo-Hidalgo PL, Garcia-Fernandez FP, RamirezPerez C. Complications associated with enteral nutrition by nasogastric tube in an internal medicine unit. J Clin Nurs 2001;10:482-90.

3. Groher ME, Crary MA. Dysphagia: clinical manage- ment in adults and children. 1st ed. Philadelphia: Mosby; 2010. p. 308-22.

4. Banfield WJ, Hurwitz AL. Esophageal stricture associated with nasogastric intubation. Arch Intern Med 1974;134:1083-6.

5. Zaninotto G, Bonavina L, Pianalto S, Fassina A, Ancona E. Esophageal strictures following nasogastric intubation. Int Surg 1986;71:100-3.

6. Nam BH, Kang YW. Endoscopic balloon dilatation for benign and malignant esophageal strictures. Korean J Intern Med 1995;48:200-6.

7. Yang US, Park SK, Kang DH, Song CS, Cho M, Song GA. The effect of bougie and balloon dilatation on benign esophageal stricture. Korean J Med 1998;54:6605.

8. Crary MA, Mann GD, Groher ME. Initial psychometric assessment of a functional oral intake scale for dysphagia in stroke patients. Arch Phys Med Rehabil 2005;86:1516-20.

9. Nagler R, Wolfsohn AW, Lowman RM, Spiro HM. Effects of gastric intubation on normal mechanisms preventing gastroesophageal reflux. N Engl J Med 1960;262:1325-8.

10. Egan JV, Baron TH, Adler DG, Davila R, Faigel DO, Gan SL, et al. Esophageal dilation. Gastrointest Endosc 2006;63:755-60. 